\title{
Processing spatially aliased arrays
}

\author{
Melvin J. Hinich \\ Virginia Polytechnic Institute and State University, Blacksburg, Virginia 24061 \\ (Received 9 September 1977; revised 13 June 1978) \begin{abstract}
than twice the distance between the closest adjacent sensors. .This penomenon, called spatial aliasing, is most pronounced when the bearing is near endfire. This paper presents a method for correcting spatial aliasing of broadband signals using the frequency-wavenumber approach to array processing. Also presented is is a method for averaging the $\omega-\kappa$ Fourier coefficients that will give a more accurate bearing estimator than the method presented by Clay, Hinich, and Shaman [J. Acoust. Soc. Am. 53, 1161-1166 (1973)]. The methods discussed in this paper can be used for a sparse unequally spaced array which is detecting a dispersive signal.
\end{abstract} \\ A linear array can detect a plane wave signal at a wrong bearing if the signal wavelengths are shorter
}

PACS numbers: 43.60.Gk

\section{INTRODUCTION}

The rule for avoiding aliasing in spectral analysis is well known by now. The sampling inteval must be less than one half the smallest period. There is a spatial analog in array theory to the time aliasing problem. In order to simplify the exposition I will begin with the simplest wave model using complex variable notation.

Let $\epsilon(t, x)$ denote the noise field observed at time $t$ and position $x$. Suppose that a sensor located at $x$ detects a single-frequency plane wave plus noise,

$$
p(t, x)=A \exp i \omega_{0}[t-(x / c) \cos \theta]+\epsilon(t, x),
$$

where $c$ is the phase velocity, $\theta$ is the direction of propagation of the wave with respect to the $x$ axis, and $A$ is the complex amplitude of the wave. The frequency in hertz is $f_{0}=\omega_{0} / 2 \pi$ 。

Delay-and-sum filtering (beamforming) is the method widely used to estimate the direction of the signal, provided that the waveguide is essentially nondispersive in the region about the array. ${ }^{1}$ Delays for each sensor channel are computed for an assumed direction, the signals are delayed and summed, and the sum is squared and integrated over the duration of the observation period. The search method is equivalent to steering the array in the direction which maximizes the energy in the main beam.

In order to explain spatial aliasing, consider a two element array whose sensors are $d \mathrm{~m}$ apart. ${ }^{2}$ Using beamforming, the bearing estimate is $\arccos \left(c d^{-1} \Delta\right)$, where $\Delta$ is the delay of one channel which maximizes the correlation between the channels. ${ }^{3}$ But from (1) it follows that the correlation of the two signals is maximized by any one of the following delays: $\Delta_{n}=(d / c) \cos \theta$ $+\left(n / f_{0}\right)$ for $n=0, \pm 1, \pm 2, \ldots$. In terms of the wavelength $\lambda_{0}=c / f_{0}$, these equivalent delays are $\Delta_{n}$ $=c^{-1} d\left(\cos \theta+n \lambda_{0} d^{-1}\right)$. At least one of these delays will have $c d^{-1} \Delta_{n}$ fall in the domain of the principal value of the in verse cosine function. Thus if $-1<\cos \theta+n \lambda_{0} d^{-1} \leq 1$, then the plane wave will appear to be on beams whose bearings are $\theta_{a}(n)=\arccos \left(\cos \theta+n \lambda_{0} d^{-1}\right)$. This will be true for any linear array with a minimum between-sensor spacing of $d \mathrm{~m}$. For example, if $d=\lambda_{0}$ and $\theta=20^{\circ}$, then $\theta_{a}=93.5^{\circ}$. Aliasing can thus be avoided for any $\theta$ if $d<\lambda_{0} / 2$, but this may not be possible in applications where the signal is broadband and $\theta$ is near $0^{\circ}$ or $180^{\circ}$. In many applications, the higher-frequency components of the signal are aliased when the source is near endfire.

It is possible to dealias the data for a broadband signal using the sample frequency-wavenumber spectrum. Let me briefly review this mathematical equivalent of beamforming. ${ }^{4}$

\section{FREQUENCY-WAVENUMBER ANALYSIS FOR BROADBAND SIGNALS}

Suppose that each sensor channel is simultaneously sampled at times $t_{j}=j \delta, j=0,1, \ldots, N-1$. The parameter $\delta$ is the sampling interval, and let $T=N \delta$ denote the sampling period. Consider the time-space Fourier transform

$$
P(\omega, \kappa)=\frac{1}{M N} \sum_{m=0}^{M-1} \sum_{j=0}^{N-1} p\left(t_{j}, x_{m}\right) \exp \left[i\left(\kappa x_{m}-\omega t_{j}\right)\right],
$$

where $x_{m}$ is the position of the $m$ th sensor in an $M$ element linear array. Counting from zero is a convention used to simplify the Fourier transform mathematics.

Let $S_{\epsilon}(\omega)$ denote the power spectrum of the noise, and assume it is spatially incoherent. Then $S_{\epsilon}(\omega)=\lim _{N \rightarrow \infty} N^{-1}$ $\times\left|\sum_{j=0}^{N-1} \epsilon\left(t_{j}, x_{m}\right) \exp \left(-i \omega t_{j}\right)\right|^{2}$. The normalized sample frequency-wavenumber spectrum is

$$
S(\omega, \kappa)=|P(\omega, \kappa)|^{2}, \quad-\pi / d \leq \kappa \leq \pi / d .
$$

This spectrum has a peak of $|A|^{2}$ at $\omega=\omega_{0}$ and $\kappa=(2 \pi /$ $\left.\lambda_{0}\right) \cos \theta$ against a background of order $(M N)^{-1} S_{\epsilon}\left(\omega_{0}\right)$ when $M N$ is large. (See Fig. 1.)

Assuming that the noise field is Gaussian, Hinich, and Shaman ${ }^{5}$ show that the maximum-likelihood estimator of $\theta$ is

$$
\hat{\theta}=\arccos \left(c \omega_{0}^{-1} \hat{\kappa}\right),
$$

where $\hat{\kappa}$ is the wavenumber which maximizes the sample $\omega-\kappa$ spectrum, i. e., $S\left(\omega_{0}, \hat{\kappa}\right)=\max _{|\kappa|<\tau / d} S\left(\omega_{0}, \kappa\right)$. There is no aliasing problem as long as $d<\lambda_{0} / 2$.

The mean square error (mse) of $\hat{\theta}$ is (approximately)

$$
\operatorname{mse}(\hat{\theta}) \simeq \frac{3}{2 M W^{2} \rho(\pi \sin \theta)^{2}}
$$




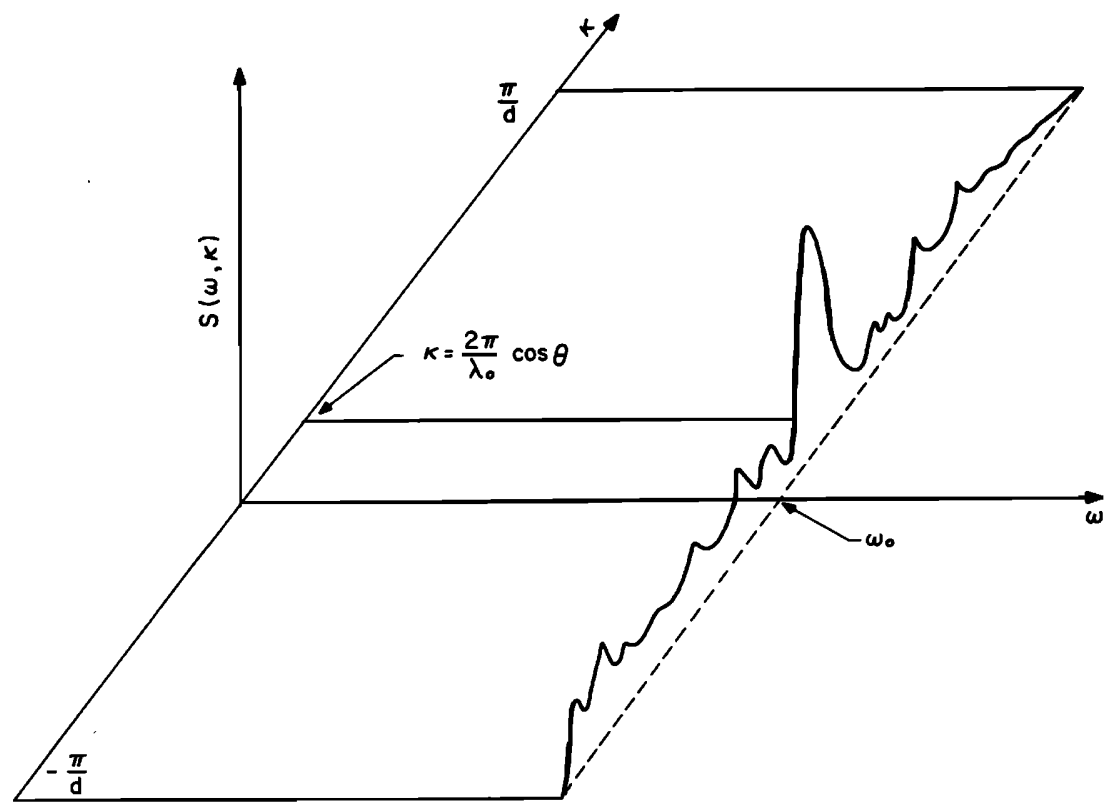

FIG. 1. Peak of sample $\omega-\kappa$ spectrum at projected wavenumber corresponding to source bearing.

for $\theta \neq 0$ or $\pi$, where $\rho=N|A|^{2} / S_{\epsilon}\left(\omega_{0}\right)$ is the energy signal-to-noise ratio in the $1 / T-\mathrm{Hz}$ band centered at $\omega_{0}$, and $W$ is the aperture of the array as measured by the number of wavelengths, i.e., $W=L / \lambda_{0}$, where $L$ is the length of the array.

When the signal has broadband energy, the frequencywavenumber spectrum is computed for each $1 / T-\mathrm{Hz}$ sub-band in the band. To be explicit, suppose that

$p(t, x)=\int_{\omega_{a}}^{\omega_{b}} A(\omega) \exp \left\{i \omega\left[t-\frac{x}{c(\omega)} \cos \theta\right]\right\} d \omega+\epsilon(t, x)$,

where the phase velocity $c(\omega)$ is expressed as a function of frequency in order to allow for the possibility of dispersion. When the propagation is significantly dispersive, beamforming wastes signal energy and gives poor results. The frequency-wavenumber approach can easily handle dispersion provided that $c(\omega)$ is a known function.

The $\omega-\kappa$ spectrum $S(\omega, \kappa)$ is computed for the $B=\left(\omega_{b}\right.$ $\left.-\omega_{a}\right) T / 2 \pi$ narrow bands of bandwidth $T^{-1} \mathrm{~Hz}$ in $\left(\omega_{a}, \omega_{b}\right)$. Let $\omega_{l}$ denote the center frequency of the $l$ th sub-band $(l=1, \ldots, B)$. When $\rho\left(\omega_{l}\right)$ is constant in the band, Clay, Hinich, and Shaman ${ }^{6}$ show that the maximum likelihood estimator (mle) of $\theta$ is $\hat{\theta}=B^{-1} \bar{\lambda} \sum_{l=1}^{B} \lambda^{-1}\left(\omega_{l}\right) \hat{\theta}\left(\omega_{l}\right)$, where $\hat{\theta}\left(\omega_{l}\right)$ is the estimator given by expression (4) for $\omega_{0}=\omega_{l}, \lambda\left(\omega_{l}\right)=2 \pi c\left(\omega_{l}\right) \omega_{l}^{-1}$ is the wavelength of the $\omega_{l}$ component, and $\bar{\lambda}=B\left[\sum_{l=1}^{B} \lambda^{-1}\left(\omega_{l}\right)\right]^{-1}$. In general, the mle of $\theta$ is

$$
\tilde{\theta}=w \sum_{l=1}^{B} \rho^{1 / 2}\left(\omega_{l}\right) \lambda^{-1}\left(\omega_{l}\right) \hat{\theta}\left(\omega_{l}\right),
$$

where $w=B\left[\sum_{l=1}^{B} \rho^{1 / 2}\left(\omega_{l}\right) \lambda^{-1}\left(\omega_{l}\right)\right]^{-1}$. The approximate mean square error of $\tilde{\theta}$ is

$$
\operatorname{mse} \tilde{\theta} \simeq 3 w^{2} / 2 M L^{2} B(\pi \sin \theta)^{2} \text {. }
$$

These results hold even when the noise field is partially coherent since the sample $\omega-\kappa$ amplitude values of a Gaussian noise field at any two points $\left(\omega_{1}, \kappa_{1}\right)$ and $\left(\omega_{2}, \kappa_{2}\right)$ are asymptotically independent as $M \rightarrow \infty$, provided that the true $\omega-\kappa$ noise spectrum is continuous. ${ }^{7}$ In other words, $P\left(\omega_{1}, \kappa_{1}\right)$ and $P\left(\omega_{2}, \kappa_{2}\right)$ are asymptotically independent as $M \rightarrow \infty$ if $\omega_{1} \neq \omega_{2}$, or $\kappa_{1} \neq \kappa_{2}$.

If the narrowband $S / N$ ratio $\rho\left(\omega_{l}\right)$ is of the order of $M^{-1}$, then the noise in the sample $\omega-\kappa$ spectrum obscures the peak at $\omega=\omega_{l}, \kappa=2 \pi \lambda^{-1}\left(\omega_{l}\right) \cos \theta$ due to the coherent signal. Consequently the estimator $\hat{\theta}$ gives a poor estimate of $\theta$ when many of the narrowband $S / N$ ratios are $O\left(M^{-1}\right)$.

There is another way to utilize the sample $\omega-\kappa$ Fourier coefficients in order to obtain a more accurate estimate of $\theta$ when $\rho\left(\omega_{j}\right)$ is $O\left(M^{-1}\right)$ for some $j$ 's. For each $l=1, \ldots, B$ and wavenumber $\kappa_{l}=2 \pi \lambda^{-1}\left(\omega_{l}\right)$, define the function

$$
\begin{aligned}
P_{l}(y) & =P\left(\omega_{l}, \kappa_{l} y\right) \\
& =\frac{1}{M N} \sum_{m=0}^{M-1} \sum_{j=0}^{N-1} p\left(t_{j}, x_{m}\right) \exp \left[i\left(\kappa_{l} y x_{m}-\omega_{l} t_{j}\right)\right],
\end{aligned}
$$

where the range of $y$ is $-\lambda\left(\omega_{l}\right) / 2 d \leq y \leq \lambda\left(\omega_{l}\right) / 2 d$. Assume that $d<\lambda\left(\omega_{b}\right) / 2 .^{8}$ The function $P_{l}(y)$, which is the time-space Fourier transform rescaled in units of $\cos \theta$, will have a peak at $y=\cos \theta$ for each $l$ as long as $M^{-1} \ll \rho\left(\omega_{l}\right)$. The average rescaled Fourier transform

$$
\bar{P}(y)=B^{-1} \sum_{i=1}^{B} P_{l}(y)
$$

has a pronounced peak at $y=\cos \theta$ when $B$ is large even if $\rho\left(\omega_{j}\right)$ is small for many $j$.

The fact that $\bar{P}(y)$ has a peak at $\cos \theta$ suggests the following alternative estimator of $\theta$ when there is no aliasing. Define $\hat{\theta}=\arccos y$ where $\hat{y}$ is the value which maximizes $|\bar{P}(y)|^{2}$ in the range $-1 \leq y \leq 1$. When $\rho$ is constant in the band, it is shown in the Appendix that the approximate mean square error of $\hat{\theta}$ for an equally 


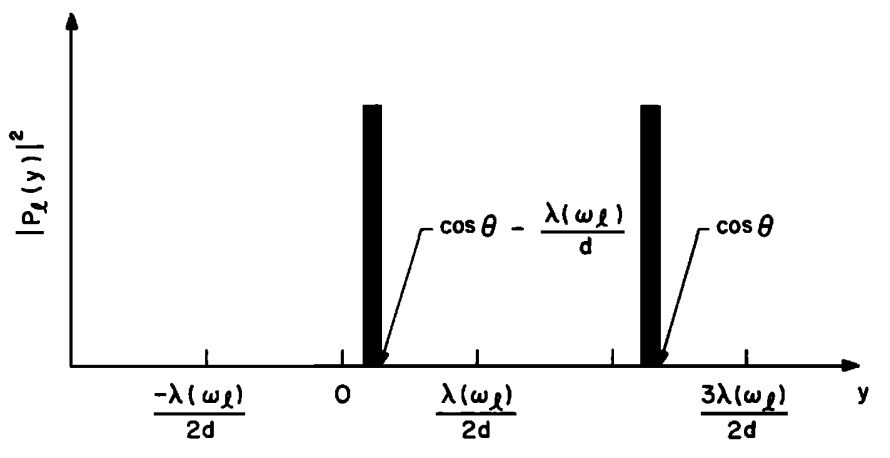

FIG. 2. Periodicity of the rescaled $\omega-\kappa$ spectrum.

spaced array is the same as that for the maximum likelihood estimator.

\section{BEARING ESTIMATION UNDER ALIASING}

Suppose that for some frequency $\omega^{*}$ in the band $\left(\omega_{a}, \omega_{b}\right), \lambda\left(\omega^{*}\right)<2 d$. Aliasing is then possible for all frequencies $\omega \geq \omega^{*}$. For example, if $\lambda\left(\omega_{1}\right) / 2 d<\cos \theta$ $<3 \lambda\left(\omega_{l}\right) / 2 d$ for a $\omega_{l} \geq \omega^{*}$, then the peak of $P_{l}(y)$ corresponding to the wavenumber $\kappa_{l}=2 \pi \lambda^{-1}\left(\omega_{l}\right)$ will be at $y=\cos \theta-\lambda\left(\omega_{l}\right) / d$. (See Fig. 2.)

The aliasing problem can be corrected. In the spirit of the beamforming bearing search, let $\theta_{0}$ denote a test value for $\theta$. Let $n_{l}$ denote the integer such that

$$
\frac{\left(2 n_{l}-1\right) \lambda\left(\omega_{l}\right)}{2 d}<\cos \theta_{0}<\frac{\left(2 n_{l}+1\right) \lambda\left(\omega_{l}\right)}{2 d},
$$

with the convention that $n_{l}=0$ if there is no aliasing at $\omega_{l}$ for $\theta_{0}$. Then compute the corrected average scaled Fourier transform

$$
\bar{P}_{c}\left(\cos \theta_{0}\right)=B^{-1} \sum_{l=1}^{B} P_{l}\left(\cos \theta_{0}-\frac{n_{l} \lambda\left(\omega_{l}\right)}{d}\right) .
$$

This computation can also be accomplished by first computing the $\omega-\kappa$ transform at $\omega_{l}$ and $\kappa=\kappa_{2} \cos \theta_{0}$ $-n_{l} 2 \pi / d$, and then scaling the $\kappa$ axis as previously shown. The statistic $\bar{P}_{c}\left(\cos \theta_{0}\right)$ is calculated for each $\theta_{0}$ on a grid of bearings whose fineness is predetermined. For most array geometries, this more complicated calculation needs to be done only for small values of $\theta_{0}$ since aliasing is generally a problem only for source bearings near endfire.

The estimate of $\theta$ is the particular bearing for which $\left|P_{c}\right|^{2}$ is a maximum. Recall that if there is no plane wave signal, the $\omega-\kappa$ spectrum and thus $\left|P_{c}\right|^{2}$, will be of the order of $(M N)^{-1} S_{\epsilon}$.

\section{APPENDIX}

Let $A\left(\omega_{l}\right)=A$ and $S_{\epsilon}\left(\omega_{l}\right)=\sigma_{\epsilon}^{2}$ in the band. If $x_{m}=m d$, then from (6) and (9)

$$
P_{l}(y)=A \frac{1-\exp \left[i \kappa_{l}(y-\cos \theta) d M\right]}{1-\exp \left[i \kappa_{l}(y-\cos \theta) d\right]}+u_{2} .
$$

For large $N$, the $u_{\imath}$ are independent zero-mean random errors with variance $E\left|u_{l}\right|^{2}=(M N)^{-1} \sigma_{\epsilon}^{2}$. When $\mid y-\cos \theta$ $\ll \lambda_{l}(2 \pi d M)^{-1}$ for each $l$,

$$
\bar{P}(y) \simeq A \frac{1-\exp [i \bar{\kappa}(y-\cos \theta) d M]}{1-\exp [i \bar{\kappa}(y-\cos \theta) d]}+\bar{u},
$$

where the error $\bar{u}=B^{-1} \sum_{l=1}^{B} u_{l}$ has variance $(M N B)^{-1} \sigma_{\epsilon}^{2}$, and $\bar{\kappa}=2 \pi \bar{\lambda}^{-1}$. Note that when there is no dispersion and thus $c$ is a constant, $\bar{\kappa}=\bar{\omega} / c$, where $\bar{\omega}=B^{-1} \sum_{l=1}^{B} \omega_{l}$. It then follows from the proof of Theorem 3.3 of Hinich and Shaman that for large $M$,

$$
E(\hat{y}-\cos \theta)^{2} \simeq 6(\bar{\kappa} d)^{-2} M^{-3}(B \rho)^{-1},
$$

where $\rho=N|A|^{2} \sigma_{\epsilon}^{-2}$. Using the Taylor series approximation of $\hat{\theta}=\arccos \hat{y}$ about $y=\cos \theta$, it follows from (A3) that

$$
\operatorname{mse}(\hat{\theta})=\frac{3 \bar{\lambda}^{2}}{2 M^{3} d^{2} B \rho(\pi \sin \theta)^{2}} .
$$

This is the same approximation as (8) when $\rho\left(\omega_{l}\right)=\rho$ for each $l$, since $L=M d$.

The rescaled spectrum $|\bar{P}(y)|^{2}$ has a peak at $y=\cos \theta$ of $|A|^{2}$ against a background of order $(M N E)^{-1} \sigma_{\epsilon}^{2}$. Thus when $B$ is large, the signal is detected using $\bar{P}$ even if $\rho \simeq M^{-1}$.

\section{ACKNOWLEDGMENT}

This work was supported by the Office of Naval Research under contract. I wish to thank Dr. G. C. Carter, Mr. Carl Bennett, and the referees for their helpful suggestions.

${ }^{1}$ M. J. Levin, "Least-Squares Array Processing for Signals of Unknown Form," Radio Electron Eng. 29, 213-222 (1965).

${ }^{2} \mathrm{~A}$ two element array is the simplest linear array. When the sensors lie on a straight line, it is impossible to identify from the data whether the direction of the wave relative to the array axis is $\theta$ or $-\theta$, i. e., there is a "left-right" ambiguity in the model. This ambiguity can be resolved using a two-dimensional (planar) array. See Ref. 6.

${ }^{3}$ G. C. Carter and C. H. Knapp, "Time Delay Estimation," Proc. IEEE Conf. Acoust. Speech Signal Process., 357-360 (1976).

${ }^{4} \mathrm{C}$. S. Clay and M. J. Hinich, "Use of a Two-Dimensional Array to Receive an Unknown Signal in a Dispersive Waveguide," J. Acoust. Soc. Am. 47, 435-440 (1970).

${ }^{5}$ M. J. Hinich and P. Shaman, "Parameter Estimation for an $R$-Dimensional Plane Wave Observed with Additive Independent Gaussian Errors," Ann. Math. Stat. 43, 153-169 (1972).

${ }^{6}$ C. S. Clay, M. J, Hinich, and P. Shaman, "Error Analysis of Velocity and Direction Measurements of Plane Waves Using Thick Large-Aperture Arrays," J. Acoust. Soc. Am. 53, 1161-1166 (1973).

'D. Brillinger, Time Series, Data Analysis and Theory (Holt, Rinehart and Winston, New York, 1975).

${ }^{8}$ The smallest wavelength in the band is $\lambda\left(\omega_{z}\right)$. As long as $d$ $<\lambda\left(\omega_{b}\right) / 2$, there is no aliasing and $|\cos \theta|<\lambda\left(\omega_{l}\right) / 2 d$ for all $l=1, \ldots, B$. 